\title{
Modified SHA-1 Algorithm
}

\author{
Rogel L. Quilala ${ }^{1}$, Ariel M. Sison², Ruji P. Medina ${ }^{3}$ \\ ${ }^{1,3}$ Technological Institute of the Philippines, 938 Aurora Blvd., Cubao, Quezon City, Philippines \\ ${ }^{2}$ Emilio Aguinaldo College, 1113-1117 San Marcelino St., Paco, Manila 1000, Philippines
}

\begin{tabular}{|c|c|}
\hline Article Info & ABSTRACT \\
\hline Article history: & Hashes are used to check the integrity of data. This paper modified SHA-1 \\
\hline Received Feb 27, 2018 & $\begin{array}{l}\text { by incorporating mixing method in every round for better diffusion. The } \\
\text { modification increased the hash output to } 192 \text {-bits. Increasing the output }\end{array}$ \\
\hline Revised Apr 21, 2018 & increases the strength because breaking the hash takes longer. Based on the \\
\hline Accepted Jun 14, 2018 & $\begin{array}{l}\text { different message types, avalanche percentage of modified SHA-1 showed } \\
\text { better diffusion at } 51.64 \% \text {, higher than the target } 50 \% \text {, while SHA-1 }\end{array}$ \\
\hline Keywords: & $\begin{array}{l}\text { achieved } 46.61 \% \text {. The average execution time noted for modified SHA- } 1 \text { is } \\
0.33 \text { seconds while SHA- } 1 \text { is } 0.08 \text { seconds. Time increases as the number of }\end{array}$ \\
\hline Hash & messages hashed increases; the difference is negligible in fewer messages. \\
\hline Data integrity & $\begin{array}{l}\text { On character hits, that is - no same character in the same position, modified } \\
\text { SHA- } 1 \text { achieved lower hit rate because of the mixing method added. The }\end{array}$ \\
\hline Security & modifications' effectiveness was also evaluated using a hash test program. \\
\hline Cryptography & After inputting 1000 hashes from random strings, the result shows no \\
\hline Avalanche & duplicate hash. \\
\hline
\end{tabular}

Copyright $\odot 2018$ Institute of Advanced Engineering and Science. All rights reserved.

\section{Corresponding Author:}

Rogel L. Quilala,

Technological Institute of the Philippines

938 Aurora Blvd., Cubao, Quezon City, Philippines.

Email: rlquilala@gmail.com

\section{INTRODUCTION}

In checking data integrity, cryptographic hash algorithms performs significant part to information security [1], [2]. Data files used hashes for verifying its integrity, where a little change will cause a different hash value [3].Hash assure that the recipient obtained the message sent by the source and that there is no form of alteration done during transmission [4]. The representation of the message in compressed form is called message digest or hash value. Hash value act as a digital fingerprint of the message or file, wherein a message can only have one distinct hash value thus no two messages should have the same hash [4]. If the hash value differs, hackers did alterations during transit resulting in the compromised integrity of the message. Electronically transmitted files, digital signature, tamper detection, password protection, and security in protocols apply hash for integrity verification [5], [6].

Seven approved hash algorithms are in Secure Hash Standard (SHS) Federal Information Processing Standards Publication (FIPS PUB 180-4) namely: SHA-1, SHA-224, SHA-256, SHA-384 SHA-512, SHA$512 / 224$, and SHA-512/256 with hash length of 160, 224, 256, 384, 512, 224 and 256 bits, respectively [7]. SHA family uses the traditional iterative structure by Merkle-Damgard (M-D) [8], [9]. Even though M-D construction ensures the security of hash functions, it suffers from some vulnerabilities due to structural weakness [10]. That is why more hash functions that address shortcomings in the M-D construction are being suggested incorporating minimal changes [11] such as wide and double pipe construction, 3C, prefix, chop, sponge, and others each exhibiting their strengths and weaknesses. In this paper, the construction will be modified by adding a counter and XORing the number to the intermediate hash value. With this additional process, the modified SHA-1 strengthened the construction because of the addition of the counter which changes at every step. 
National Institute of Standards and Technology (NIST) published Secure Hash Algorithm 1 (SHA-1) as a cryptographic hash function [7], [12]. SHA-1 produces 160-bit hash value and is considered fast [13]. It is the most widely used hash algorithm in a vast range of applications such as Digital Signatures, TLS/SSL, SSH and PGP [14]-[16] due to its time efficiency and robustness [17]. At present, $21 \%$ of websites in the world still use SHA-1 in signing certificates [18]. SHA-1 based fingerprint is used widely and supported for verification [19].

Other hash functions also exist such as MD5 by Ronald L. Rivest released in 1992 that can compress any data length to a hash value of 128bits [20], but real collision broke MD5 entirely in 2004 [21], [22]. SHA-0 in 1993 is an MD4 hash function used for authentication, is believed to be not safe after several successful collision attacks in 2004 and 2005 [1]. SHA-2 and SHA-3 provide more extended hash value that is more complicated to break [11], [13], but they are more complex and not as time efficient as SHA-1 [14] [23], [24]. The increased number of rounds in SHA3 makes it less susceptible to collision resistance and preimage resistance attacks when measured against SHA2, MD5, and SHA1 and others [25] but the use of a sponge function construction can be considered neither as an advantage nor a disadvantage because this function is a new construction that is not yet very well analyzed [26].

Though SHA-1 is popular, widely used and accepted as standard by NIST. Some noted that it does not seem to offer sufficient avalanche effect with regards to the distribution of the input differences, while other noted some unexpected weaknesses in the construction of all the step updating functions [1], [27]. This problem will lead to the possibility of having two different input that will yield the same output value in the middle of algorithm or compression function [20] [28]. Therefore, it is necessary to design a function with better diffusion to spread the output in each round and prevent the same output in the next coming stages [20], [29]-[30].

Several studies made several enhancements on SHA-1 to attain additional diffusion [31], [32] but did not show the bit-difference on the simulation of result or have shown lower bit difference. One study has added the MD5 hash to SHA-1 [29] that indicates that the bit-difference of SHA-192 is lower than SHA-160. This approach might suffer from the same weakness as that of MD5 [21], [22]. Others have not included the actual message in the comparison of bit-difference. [23]. Therefore, the researcher has decided to improve SHA-1 algorithm by increasing hash size output from 160 to 192 bits and provide better diffusion. Another enhancement of SHA-1 makes use of 320-bit hash by doubling the message digest size and hash size [14]. This enhancement decreases the chances of the collision, but this approach requires more processing time since it makes use of a higher block size. Notice that all enhancements made on SHA 1 uses the chaining variables $\mathrm{A}, \mathrm{C}$, and $\mathrm{D}$ in each round as is and is just shifted to the next chaining variables and sends it to the next round. From here, the researcher proposed to devise the mixing method to diffuse variables A, C, and D better for each iteration.

This study intended to modify SHA-1 algorithm by increasing the output to 192-bits and strengthening the hash function by adjusting the compression function through the incorporation of additional mixing method in every round with the intention of attaining better diffusion. The objectives of this study are to evaluate the performance of the modified SHA-1 through avalanche effect and to test the modified SHA-1 algorithm regarding time and message complexity.

The main impact of this work is the improvement of SHA-1 by introducing additional mixing method in every round to achieve better diffusion characteristics. The study will contribute to the improvement of the compression function used by SHA1 by increasing the output of the hash value to 192bits to strengthen the algorithm. Higher time will be needed to break the hash.

\section{RESEARCH METHOD}

\subsection{Research Procedure}

Figure 1 shows the proposed modified SHA-1 construction with the counter. An added counter was XORed to the intermediate hash value. The addition of this process strengthened the M-D construction because of a number assigned to the counter that changes in every step. The counter will start at an initial value of zero and is incremented by 1 for every message block until the last block.

The proposed SHA algorithm of the compression function retained the eighty rounds. The modified SHA-1 increased the message digest from 160-bits to 192-bits to strengthen the algorithm. To achieve this, one additional chaining variables $F$ was added. Next, F was XORed to the output of E before going to A. All researchers have used variables $\mathrm{A}, \mathrm{C}$, and $\mathrm{D}$ as is. In every round, these variables were injected into the mixing function to achieve better diffusion. The variables are mixed every round and send it to the next round. This mixing function guarantees that the input values will spread out thus promoting good diffusion in each round because the contents of the variables will not be the same in the coming rounds. Variable E goes to variable $\mathrm{F}$ after own addition operations. Figure 2 shows the proposed modification on SHA-1 with the 
added mixing method. In the proposed hash algorithm, we note significant changes in the elementary function.

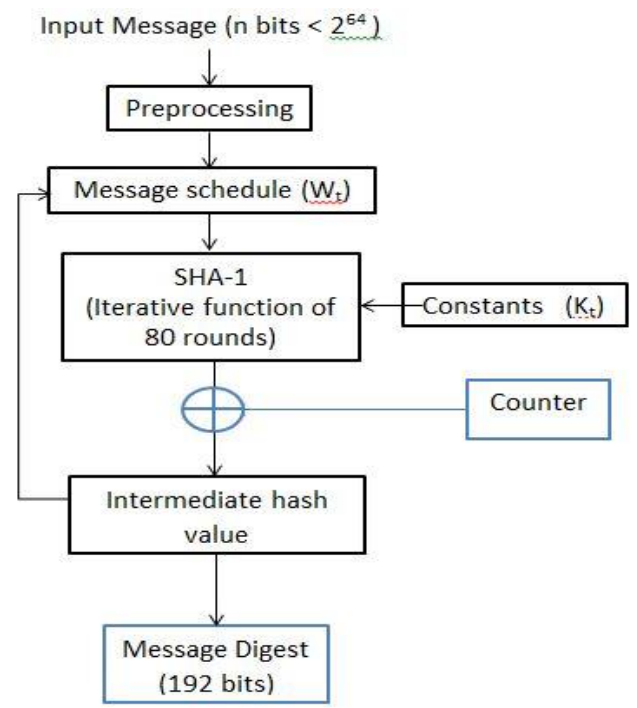

Figure 1. Proposed modification on SHA-1 construction

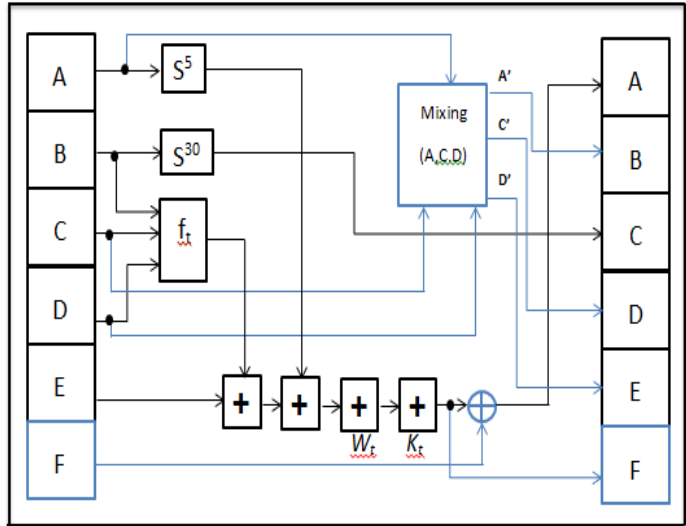

Figure 2. Proposed modification on SHA-1 compression with added mixing method

The modified SHA-1 follows the same step in SHA except for the computation of the message digest. The padded message is used to compute for the message digest. The computation uses two buffers (A, $\mathrm{B}, \mathrm{C}, \mathrm{D}, \mathrm{E}, \mathrm{F}$ and $\mathrm{H}_{0}, \mathrm{H}_{1}, \mathrm{H}_{2}, \mathrm{H}_{3}, \mathrm{H}_{4}, \mathrm{H}_{5}$ ). The first buffer uses five 32-bit words, and the second buffer comprises of eighty 32-bit words (W0, W1 ... W79). This process also uses TEMP1 and TEMP2 buffers. $\{\mathrm{Hj}\}$ are initialized before processing any blocks with values of 67452301, EFCDAB89, 98BADCFE, 10325476, C3D2E1F0, $40385172\left(\mathrm{H}_{1}-\mathrm{H}_{5}\right)$. Let hash value length be $\mathrm{m}$.

Modified SHA-1 steps to process the message in 16-word blocks:

a) Split Mi into 16 words starting from left to right, $\mathrm{W}_{0}, \ldots \mathrm{W}_{15}$

b) When $\mathrm{t}=16$ to 79 , we do $\mathrm{W}_{\mathrm{t}}=\mathrm{S}^{1}\left(\mathrm{~W}_{\mathrm{t}-3} \mathrm{XOR} \mathrm{W}_{\mathrm{t}-8} \mathrm{XOR} \mathrm{W}_{\mathrm{t}-14} \mathrm{XOR} \mathrm{W}_{\mathrm{t}-16}\right)$.

c) Then let $\mathrm{A}=\mathrm{H}_{0}, \mathrm{~B}=\mathrm{H}_{1}$, until $\mathrm{F}=\mathrm{H}_{5}$, counter $=\mathrm{m}$

d) When $\mathrm{t}=0$ upto 79 do

mixed $\mathrm{ACD}=\operatorname{mixing} \mathrm{ACD}(\mathrm{A}, \mathrm{C}, \mathrm{D})$ 
$\mathrm{A}^{\prime}=$ mixedACD; $\mathrm{C}^{\prime}=$ mixed $\mathrm{ACD} ; \mathrm{D}^{\prime}=\operatorname{mixed} \mathrm{ACd}$

TEMP1 $=S^{5}(A)+f_{t}(B, C, D)+E+W_{t}+K_{t}$;

TEMP2 $=\mathrm{F}$ xor TEMP1

$\mathrm{E}=\mathrm{D}^{\prime} ; \mathrm{D}=\mathrm{C}^{\prime} ; \mathrm{C}=\mathrm{S}^{30}(\mathrm{~B}) ; \mathrm{B}=\mathrm{A}^{\prime} ; \mathrm{A}=\mathrm{TEMP} 2 ; \mathrm{F}=\mathrm{TEMP} 1$

e) counter $+=m$, then do $\mathrm{H}_{0}=\left(\mathrm{H}_{0}+\mathrm{A}\right)$ xor counter, $\mathrm{H}_{1}=\left(\mathrm{H}_{1}+\mathrm{B}\right)$ xor counter, $\mathrm{H}_{2}=\left(\mathrm{H}_{2}+\mathrm{C}\right)$ xor counter, $\mathrm{H}_{3}=\left(\mathrm{H}_{3}+\mathrm{D}\right)$ xor counter, $\mathrm{H}_{4}=\left(\mathrm{H}_{4}+\mathrm{E}\right)$ xor counter, $\mathrm{H}_{5}=\left(\mathrm{H}_{5}+\mathrm{F}\right)$ xor counter.

After processing $\mathrm{M}_{\mathrm{n}}$, these words represent the computed 192-bit hash value:

$\mathrm{H}_{0} \mathrm{H}_{1} \mathrm{H}_{2} \mathrm{H}_{3} \mathrm{H}_{4} \mathrm{H}_{5}$

The purpose of the Mixing (A, C, D) function is to accept the working variables A, C, and D as the input column then spread the bits out to different places in the output column A', C,' and D'. The mix is arranged from right to left in row-wise fashion as illustrated in Figure 3.

\begin{tabular}{|c|c|c|c|c|c|c|}
\hline A & C & D & \multirow{2}{*}{ Mixing $(A, C, D)$} & \multirow[t]{2}{*}{$A^{\prime}$} & \multirow[t]{2}{*}{$\mathbf{C}^{\prime}$} & \multirow[t]{2}{*}{ D' } \\
\hline$A_{0,0}$ & $\mathrm{C}_{0,1}$ & $\mathrm{D}_{0,2}$ & & & & \\
\hline$A_{1,0}$ & $\mathrm{C}_{1,1}$ & $D_{1,2}$ & \multirow{5}{*}{$K$} & $A_{2,0}$ & $A_{1,0}$ & $A_{0,0}$ \\
\hline$A_{2,0}$ & $\mathrm{C}_{2,1}$ & $\mathrm{D}_{2,2}$ & & & & 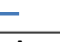 \\
\hline \multirow[t]{3}{*}{$A_{3,0}$} & $\mathrm{C}_{3,1}$ & $D_{3,2}$ & & $\mathrm{C}_{1,1}$ & $\mathrm{C}_{0,1}$ & $A_{3,0}$ \\
\hline & & & & $\mathrm{D}_{0,2}$ & $\mathrm{C}_{3,1}$ & $\mathrm{C}_{2,1}$ \\
\hline & & & & $\mathrm{D}_{3,2}$ & $\mathrm{D}_{2,2}$ & $\mathrm{D}_{1,2}$ \\
\hline
\end{tabular}

Figure 3. Mixing function

\subsection{Evaluation Metrics} complexity.

The performance of the modified SHA-1 was evaluated through avalanche effect, time and message

Avalanche effect is a suitable characteristic in a hash function which indicates that a change in the input bit of the hash results to a difference on the probability of the output bit. If the chance is close to $50 \%$, the hash function is considered good. A 50\% avalanche percentage shows that the difference of the output hash value and the input change is at least half and a probability higher than $50 \%$ displays improved statistical performance [33].

Time notes the speed to generate the hash in seconds. Classification of the message type is two message with 1-bit change, 24 messages with a difference in a few bits, two messages with distinction in the last few bits, length difference, and random strings. Performance of the hash function is also measured by comparing hash values with each other and then counting characters located at the same location with the same content [34], in this study referred to as character hit.

\section{RESULTS AND ANALYSIS}

For performance analysis, we consider different messages during the testing and time, and avalanche effect was noted for each test. The first message type is a 1-bit change in the message input. Consider the two message: Message 1: "The quick brown fox jumps over the lazy dog" and Message 2: "The quick brown fox jumps over the lazy mog".

The second message type was tested using an input with a difference in only a few bits. Table 1 lists the twenty-four messages used. The researcher inserts different characters at the beginning, middle, and last.

For the third message, consider the two words: "abc123_owlstead_1255" and “abc123_owlstead_59131".

The fourth message input is the length differences, that is the message "a a a" has a length of 5 versus message "a a" which has a length of 3 . The length of message considered was listed in Table 2 
Table 1. Message inputs with a difference of a few bits

\begin{tabular}{|c|c|}
\hline No. & Message Input \\
\hline 1 & @ AAAAAAAAAAAAAAAAAAAAAAAAAAAAAAAAAAAAAAAAAAAAAAAAAAAAAAAAAAAAAAA \\
\hline 2 & CAAAAAAAAAAAAAAAAAAAAAAAAAAAAAAAAAAAAAAAAAAAAAAAAAAAAAAAAAAAAAAA \\
\hline 3 & EAAAAAAAAAAAAAAAAAAAAAAAAAAAAAAAAAAAAAAAAAAAAAAAAAAAAAAAAAAAAAAA \\
\hline 4 & IAAAAAAAAAAAAAAAAAAAAAAAAAAAAAAAAAAAAAAAAAAAAAAAAAAAAAAAAAAAAAAA \\
\hline $\begin{array}{l}5 \\
6\end{array}$ & $\begin{array}{l}\text { QAAAAAAAAAAAAAAAAAAAAAAAAAAAAAAAAAAAAAAAAAAAAAAAAAAAAAAAAAAAAAAA } \\
\text { aAAAAAAAAAAAAAAAAAAAAAAAAAAAAAAAAAAAAAAAAAAAAAAAAAAAAAAAAAAAAAAA }\end{array}$ \\
\hline 7 & AAAAAAAAAAAAAAAAAAAAAAAAAAAAAAAAAAAAAAAAAAAAAAAAAAAAAAAAAAAAAAA \\
\hline 8 & ÁAAAAAAAAAAAAAAAAAAAAAAAAAAAAAAAAAAAAAAAAAAAAAAAAAAAAAAAAAAAAAAA \\
\hline 9 & AAAAAAAAAAAAAAAAAAAAAAAAAAAAAAAAAAAAAAAAAAAAAAAAAAAAAAAAAAAAAAAA \\
\hline 10 & AAAAAAAAAAAAAAAAAAAAAAAAAAAAAAAA@AAAAAAAAAAAAAAAAAAAAAAAAAAAAAAA \\
\hline 11 & AAAAAAAAAAAAAAAAAAAAAAAAAAAAAAAACAAAAAAAAAAAAAAAAAA \\
\hline 12 & AAAAAAAAAAAAAAAAAAAAAAAAAAAAAAAAEAAAAAAAAAAAAAAAAAAAAAAAAAAAAAAA \\
\hline 13 & AAAAAAAAAAAAAAAAAAAAAAAAAAAAAAAAIAAAAAAAAAAAAAAAAAAAAAAAAAAAAAAA \\
\hline 14 & AAAAAAAAAAAAAAAAAAAAAAAAAAAAAAAAQAAAAAAAAAAAAAAAAAAAAAAAAAAAAAAA \\
\hline 15 & AAAAAAAAAAAAAAAAAAAAAAAAAAAAAAAAaAAAAAAAAAAAAAAAAAAAAAAAAAAAAAAA \\
\hline 16 & AAAAAAAAAAAAAAAAAAAAAAAAAAAAAAAAAAAAAAAAAAAAAAAAAAAAAAAAAAAAAAA \\
\hline 17 & AAAAAAAAAAAAAAAAAAAAAAAAAAAAAAAAAAAAAAAAAAAAAAAAAAAAAAAAAAAAAAA@ \\
\hline 18 & AAAAAAAAAAAAAAAAAAAAAAAAAAAAAAAAAAAAAAAAAAAAAAAAAAAAAAAAAAAAAAAC \\
\hline 19 & \\
\hline 20 & AAAAAAAAAAAAAAAAAAAAAAAAAAAAAAAAAAAAAAAAAAAAAAAAAAAAAAAAAAAAAAAI \\
\hline 21 & AAAAAAAAAAAAAAAAAAAAAAAAAAAAAAAAAAAAAAAAAAAAAAAAAAAAAAAAAAAAAAAQ \\
\hline 22 & AAAAAAAAAAAAAAAAAAAAAAAAAAAAAAAAAAAAAAAAAAAAAAAAAAAAAAAAAAAAAAAa \\
\hline 23 & AAAAAAAAAAAAAAAAAAAAAAAAAAAAAAAAAAAAAAAAAAAAAAAAAAAAAAAAAAAAAAA \\
\hline 24 & AAAAAAAAAAAAAAAAAAAAAAAAAAAAAAAAAAAAAAAAAAAAAAAAAAAAAAAAAAAAAAAÁ \\
\hline
\end{tabular}

Table 2. Message inputs with different length

\begin{tabular}{cl}
\hline No. & Message Input \\
\hline 1 & a \\
2 & a a \\
3 & a a a \\
& \\
23 & a a a a a a a a a a a a a a a a a a \\
24 & a a a a a a a a a a a a a a a a
\end{tabular}

The fourth type is a random string of message. For this test, the message consists of characters a...z, A...Z, and 0...9. An online tool helps generate hashes from 500 random strings each of length 64 [35].

Table 3. Summary of results

\begin{tabular}{llcccc}
\hline & \multirow{2}{*}{ Message Type } & \multicolumn{2}{c}{$\begin{array}{c}\text { Avalanche } \\
(\%)\end{array}$} & \multicolumn{2}{c}{ Time } \\
& & (seconds) \\
& & MSHA-1 & SHA-1 & MSHA-1 & SHA-1 \\
\hline 1 & Two messages with 1-bit change & 56.77 & 46.25 & 0.02 & 0.02 \\
2 & 24 messages w/diff. in a few bits & 50.09 & 48.37 & 0.09 & 0.05 \\
3 & Two messages w/diff. in last few bits & 50.00 & 38.75 & 0.02 & 0.02 \\
4 & Length difference & 51.13 & 49.76 & 0.06 & 0.05 \\
5 & Random strings & 50.19 & 49.90 & 1.28 & 0.28 \\
& $\quad$ Average (\%) & 51.64 & 46.61 & 0.33 & 0.08 \\
\hline
\end{tabular}

For message type 1, the proposed modified SHA-1 achieved $56.77 \%$ while SHA-1 obtained $46.25 \%$. Hashing time for both tests is 0.02 seconds. For message type 2, avalanche effect of the proposed modification on SHA-1 obtained $50.09 \%$. The original SHA-1 attained $48.37 \%$, slightly lower than the desired 50\%. Concerning the execution time, as reflected in Table 3, it took the modified SHA-1 0.09 seconds to complete while SHA-1 took 0.05 seconds. The modified SHA-1 is a bit higher. For message type 3, the modified SHA-1 achieved exactly 50.00\% while SHA-1 got 38.75\%, which is significantly lower. Hashing time for both tests is 0.02 seconds. For message type 4, the modified SHA-1 achieved $51.13 \%$ while SHA-1 attained $49.76 \%$. The hashing time shows 0.06 and 0.05 for modified SHA and SHA-1. There is a very minimal difference when it comes to the hashing time. Lastly, for message type 5, the modified SHA achieved 50.19\% while SHA-1 arrived at 49.90\%5. The modified SHA-1 hashed the random string of message in 1.28 seconds while SHA-1 produced the hash in 0.28 . A difference of 1.00 seconds can be noted. 
Based on the average, the avalanche effect of all has increased due to the modifications made. The testing showed better diffusion result because out of the five different message types, the average avalanche percentage of modified SHA-1 was $51.64 \%$ which is higher than the target $50 \%$ while SHA achieved only 46.61\%. Regarding the time it takes to produce the hash, the time recorded was the same for two-message comparisons. An increase in time appears as the number of the message to be hashed enlarges. The average time noted for modified SHA-1 is 0.33 while SHA-1 is 0.08 . The increment is mostly due to the added mixing method and XOR operation. Although the time associated with hashing a message using modified SHA is a bit higher, there is evident character hits as shown in Table 4.

Table 4. Summary of character hits

\begin{tabular}{llcccc}
\hline & \multirow{2}{*}{ Message Type } & \multicolumn{2}{c}{ Total Character Hits } & \multicolumn{2}{c}{ Max No. Of Equal Character Hits } \\
& MSHA-1 & SHA-1 & MSHA-1 & SHA-1 \\
\hline 1 & 1-bit change & 0 & 0 & 0 & 0 \\
2 & 24 messages w/diff. in a few bits & 0 & 2 & 0 & 1 \\
3 & Two messages w/diff. in last few bits & 0 & 5 & 0 & 5 \\
4 & Length difference & 1 & 6 & 1 & 2 \\
5 & Random strings & 38 & 48 & 1 & 2 \\
\multicolumn{2}{c}{ Average (\%) } & $(2: 5)$ & $(4: 5)$ & & \\
\hline
\end{tabular}

In the modified SHA-1, message types 1,2, and three doesn't have any character hits. For message type 4 , out of the 24 hashes generated, there was one instance where the same character was at the same position. For message type 5, out of the 500 random messages, 38 hash pairs contains one character hit.

Character hits are noted more frequently in SHA-1. For message type 2, there were two hits recorded. There were five hits observed for message type 3 and the number of characters per hit ranges from 1-5 characters per hash. For message type 4, 6-character hits and the number of characters that match ranges from 1-2 per hash. For message 5, there were 44 hash pairs containing one character hit per hash and two hash pairs with 2 character hits for a total of 48 hits. Notice that the hits for the original SHA-1 are higher compared to the adjusted version.

The modified SHA-1 simulation indicates that out of the five message types, there were two instances where a character hit was noted $(2: 5$ or $40 \%)$ while in SHA-1, character hits occur 4 out of the five different message types $(4: 5$ or $80 \%)$. When considering the number of hits, the modified SHA-1 has a much lower hit rate compared to the original SHA-1 on all tests made and on all test cases.

\begin{tabular}{|r|l|}
\hline 1 & $934 f 46412 d b d e a 31 a 1 d 91 a a 27 b 19835014 a c 7 c 55$ \\
\hline 2 & a66aaff13b6012bf7bcdd8815e8d41f25ca72168 \\
\hline 3 & $33660742 c 1 e 6 f f 3 a 86489535474613 c 2 f d 0935 b 8$ \\
\hline 4 & $2458 c d 342 a b 44954 a 261 a 44 f 94507 d c 1412262 a c$ \\
\hline 5 & $7 d b 41 c 9 d 2 d 390684 b 428680 d 1475 b 4 e 2 a b 854 e 08$ \\
\hline 6 & f082a212742687a630ff4677c46f15f627490098 \\
\hline 7 & $4547 c 3 b 7 c 7 d 43 b 64 d e b e 62 b 01767284901046 a a 2$ \\
\hline 8 & f2d40a511fa54b686b7e0162529be09819571225 \\
\hline 9 & $30 b 86 e 44 e 6001403827 a 62 c 58 b 08893 e 77 c f 121 f$ \\
\hline 2 & $\ldots$ \\
\hline 23 & a320100894f91165b624376e4c8d01cea4637424 \\
\hline 24 & d091c57996091b918aaa76233bac434609734d24 \\
\hline
\end{tabular}

Figure 4. Hash list for a message with the difference in a few bits

To understand character hits, using the 24 message inputs and their hash value in SHA-1, the researcher count the values that have the same hexadecimal value at the same position. Two hexadecimal value is equal to 1 hit. Using traditional SHA-1 as shown in Figure 4, the hash of the message having a difference in a few bits found two hits (Hash 8 and 9, hash 23 and 24). Figure 5 illustrates another example using two messages with the difference in a few bits. In the modified SHA-1, the computed hash found no 
values on the same location. In SHA-1, there are nine hexadecimal values or 5 ASCII characters located at the same place.

\begin{tabular}{|c|}
\hline SHA-1 Hash value \\
\hline $992156 \mathrm{e} 04 \mathrm{e} 6511 \mathrm{eb} 30830 \mathrm{a} 8 \mathrm{dbd} 9 \mathrm{df}$ ac852e 38117 \\
\hline $992156 \mathrm{e} 0429 \mathrm{ebl}$ ebe f 46681 af $\mathrm{f} 430 \mathrm{dc} 30 \mathrm{cf} 24 \mathrm{db} 5$ \\
\hline Modified SHA-1 with mixing method hash value \\
\hline $871 \mathrm{c} 0 \mathrm{f} 73015 \mathrm{f} 30 \mathrm{ab} 2 \mathrm{e} 460896621 \mathrm{cdde} 1 \mathrm{f} 71 \mathrm{~d} 43 \mathrm{df0f} 7 \mathrm{db022}$ \\
\hline a7ef3e81 30bdbf c436ea902b9808b5a5e92688 f60106270c \\
\hline
\end{tabular}

Figure 5. Hash list of two messages with the difference in a few bits

The hash value produced by the modified hash was also tested using a hash function testing program [36]. This program takes hash values and counts how many duplicates the hash function produces. 1000 hashes from random strings were generated using the modified SHA-1 algorithm, and after running the hash test, modified SHA-1 found no duplicates. Figure 6 shows the screenshot of the hash function test.

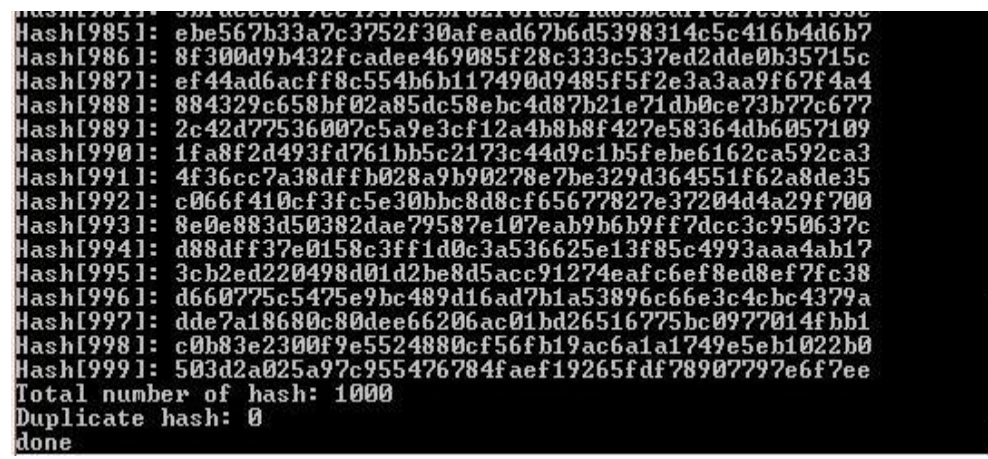

Figure 6. Hash function test

\section{CONCLUSION}

This study intended to modify SHA-1 algorithm by increasing the output to 192-bits and strengthening the hash function by adjusting the compression function through the incorporation of additional mixing method in every round with the intention of attaining better diffusion. Looking at the results of the tests done, the modified SHA-1 have better diffusion compared to the original SHA-1. The diffusion is evident by the increase in the avalanche percentage. There is an increase in the avalanche percentage although the time also increased when messages increased. The additional mixing method and XOR operation contribute to the increment in time. It is also evident that the number of hits using the modified SHA-1 was minimal or lower compared to the original SHA-1 leading to no collision. Upon using the hash function testing program, the hash values found have no duplicates. Based on the results, the modified SHA-1 can be used to test the integrity of messages. Further improvement is suggested to minimize the time consumed by the modified SHA-1 hash by studying the effect of lessening the number of rounds.

\section{REFERENCES}

[1] N. Kishore and B. Kapoor, "Attacks on and advances in secure hash algorithms," IAENG Int. J. Comput. Sci., vol. 43, no. 3, pp. 326-335, 2016.

[2] M. A. Alahmad, "Design of a New Cryptographic Hash Function - Titanium," Indones. J. Electr. Eng. Comput. Sci., vol. 10, no. 2, pp. 827-832, 2018.

[3] I. Alsmadi and M. Zarour, "Online integrity and authentication checking for Quran electronic versions," Appl. Comput. Informatics, vol. 13, no. 1, pp. 38-46, 2017.

[4] R. P. Arya, U. Mishra, and A. Bansa, "A Survey on Recent Cryptographic Hash Function Designs," Int. J. Emerg. Trends Technol. Comput. Sci., vol. 2, no. 1, pp. 117-122, 2013. 
[5] W. Chankasame and W. San-Um, "A chaos-based keyed hash function for secure protocol and messege authentication in mobile ad hoc wireless networks," Proc. 2015 Sci. Inf. Conf. SAI 2015, pp. 1357-1364, 2015.

[6] R. K. Ibraheem, R. A. J. Kadhim, and A. S. H. Alkhalid, "Anti-collision enhancement of a SHA-1 digest using AES encryption by LABVIEW,” 2015 World Congr. Inf. Technol. Comput. Appl., pp. 1-6, 2015.

[7] Q. H. Dang, "Secure Hash Standard," Gaithersburg, MD, Jul. 2015.

[8] R. C. Merkle, "One Way Hash Functions and DES," in Proceedings of the 9th Annual International Cryptology Conference on Advances in Cryptology, 1990, pp. 428-446.

[9] I. B. Damgård, “A Design Principle for Hash Functions,” CRYPTO`89 Proceedings, vol. 435. pp. 416-424, 1990.

[10] H. Tiwari and K. Asawa, "Building a 256-bit hash function on a stronger MD variant," Open Comput. Sci., vol. 4, no. 2, pp. 67-85, Jan. 2014.

[11] R. Sobti and G. Geetha, "Cryptographic hash functions: a review," IJCSI Int. J. Comput. Sci. Issues, vol. 9, no. 2, pp. 461-479, 2012.

[12] NIST, "FIPS 180-1 - Secure Hash Standard," FIPS PUB 180-1, no. April 17, 1995.

[13] P. Garg and N. Tiwari, "Performance Analysis of SHA Algorithms ( SHA-1 and SHA-192 ): A Review," Int. J., vol. 2, no. 3, pp. 130-132, 2012.

[14] S. Rao, "Advanced SHA-1 Algorithm Ensuring Stronger Data Integrity," Int. J. Comput. Appl., vol. 130, no. 8, pp. 25-27, 2015.

[15] R. A. N. Karthik, A.K. Parvathy, "Non-convex Economic Load Dispatch using Cuckoo Search Algorith," Indones. J. Electr. Eng. Comput. Sci., vol. 5, no. 1, pp. 48-57, 2017.

[16] T. Mantoro and A. Zakariya, "Securing E-mail Communication Using Hybrid Cryptosystem on Android-based Mobile Devices," TELKOMNIKA (Telecommunication, Comput. Electron. Control., vol. 10, no. 4, pp. 827-834, 2012.

[17] K. Saravanan and A. Senthilkumar, "Theoretical Survey on Secure Hash Functions and issues," Int. J. Eng. Res. Technol., vol. 2, no. 10, pp. 1150-1153, 2013.

[18] Venafi, "Venafi Research: Twenty-One Percent of Websites Are Still Using Insecure SHA-1 Certificates and Putting Users at Risk," Venafi Press Release, 2017.

[19] M. Stevens and D. Shumow, "Speeding up detection of SHA-1 collision attacks using unavoidable attack conditions.," USENIX Secur., vol. 2017, p. 173, 2017.

[20] A. Kumarkasgar, J. Agrawal, and S. Shahu, "New modified 256-bit MD5 Algorithm with SHA Compression Function," Int. J. Comput. Appl., vol. 42, no. 12, pp. 47-51, Mar. 2012.

[21] X. Wang, D. Feng, X. Lai, and H. Yu, "Collisions for Hash Functions MD4, MD5, HAVAL-128 and RIPEMD.," IACR Cryptol. ePrint Arch., vol. 5, no. October, pp. 5-8, 2004.

[22] X. Wang and H. Yu, "How to Break MD5 and Other Hash Functions," Adv. Cryptol. - EUROCRYPT 2005, pp. 19$35,2005$.

[23] S. Verma and G. S. Prajapati, "Robustness and security enhancement of SHA with modified message digest and larger bit difference," in 2016 Symposium on Colossal Data Analysis and Networking (CDAN), 2016, pp. 1-5.

[24] K. kumar Raghuvanshi, P. Khurana, and P. Bindal, "Study and Comparative Analysis of Different Hash Algorithm," J. Eng. Comput. Appl. Sci., vol. 3, no. 9, pp. 1-3, 2014.

[25] J. Sharma and D. Koppad, "Low power and pipelined secure hashing algorithm-3(SHA-3)," in 2016 IEEE Annual India Conference (INDICON), 2016, vol. 3, pp. 1-5.

[26] A. Breust and F. Etcheverry, "Why not SHA-3?," pp. 1-13, 2013.

[27] X. Wang, Y. L. Yin, and H. Yu, "Finding Collisions in the Full SHA-1," Adv. Cryptol. - CRYPTO 2005, no. 90304009, pp. 17-36, 2005.

[28] P. Karpman, T. Peyrin, and M. Stevens, "Practical Free-Start Collision Attacks on 76-step SHA-1," vol. 2012, 2015.

[29] G. Gupta and S. Sharma, "Enhanced SHA-192 algorithm with larger bit difference," Proc. - 2013 Int. Conf. Commun. Syst. Netw. Technol. CSNT 2013, pp. 152-156, 2013.

[30] X. Xu, Q. Zhao, and C. Li, "Advanced framework for iterative hash functions," Proc. - 2012 Int. Conf. Comput. Sci. Electron. Eng. ICCSEE 2012, vol. 2, pp. 599-602, 2012.

[31] C. C. G. San Jose, B. T. Tanguilig III, and B. D. Gerardo, "Enhanced SHA-1 on Parsing Method and Message Digest Formula," pp. 1-9, 2015.

[32] L. Thulasmani and M. Madheswaran, "Security and Robustness Enhancement of Existing Hash Algorithm," 2009 Int. Conf. Signal Process. Syst., pp. 253-257, 2009.

[33] M. Asgari Chenaghlu, S. Jamali, and N. Nikzad Khasmakhi, "A novel keyed parallel hashing scheme based on a new chaotic system," Chaos, Solitons \& Fractals, vol. 87, pp. 216-225, Jun. 2016.

[34] S. Deng, Y. Li, and D. Xiao, "Analysis and improvement of a chaos-based Hash function construction," Commun. Nonlinear Sci. Numer. Simul., vol. 15, no. 5, pp. 1338-1347, 2010.

[35] "Text Mechanic - Random String Generator Tool." [Online]. Available: http://textmechanic.com/texttools/randomization-tools/random-string-generator/.

[36] N. Smudge, "Hash Function Test Program," 2008. [Online]. Available: http://nickmudge.info/?post=84. 